\section{Die Goldgewinnung in den letzten zwanzig Jahren.}

Von Fritz K RULL, Ingenieur-Chemiker, Paris.

Die Goldproduktion der Erde zeigt in den letzten 20 Jahren eine ganz ungeheure Zunahme, wie dies aus der nachfolgenden, im ,New-Yorker Commercial and Financial Chronicle" veröffentlichten Tabelle hervorgeht.

\begin{tabular}{c|c|c|c}
\hline $\begin{array}{c}\text { Jahres. } \\
\text { produktion }\end{array}$ & Gewicht in kg & $\begin{array}{c}\text { Wert in Mill. M } \\
\text { (a) }\end{array}$ & $\begin{array}{c}\% \\
\text { (b) }\end{array}$ \\
\hline \hline $1851-55$ \\
$1856-60$ \\
$1861-65$ \\
$1866-70$ \\
$1871-75$ & 213752,3 & 606,82 & 100 \\
$1876-80$ & 203667,3 & 568,68 & 95 \\
$1881-85$ & 180906,9 & 504,84 & 85 \\
$1886-90$ & 190714,4 & 532,56 & 90 \\
$1891-95$ & 174324,9 & 486,78 & 82 \\
1896 & 163891,1 & 457,38 & 77 \\
1897 & 152966,9 & 426,72 & 72 \\
1898 & 165477,9 & 462,00 & 78 \\
1899 & 244511,3 & 682,50 & 115 \\
1900 & 305404,3 & 853,60 & 143 \\
1901 & 357143,4 & 997,08 & 167 \\
1902 & 435909,2 & 1216,74 & 204 \\
1903 & 473350,2 & 1321,32 & 222 \\
1904 & 494502,1 & 1101,24 & 185 \\
& 449010 & 1119,72 & 188 \\
& 490696,2 & 1253,70 & 211 \\
& 526402 & 1370,04 & 230 \\
\hline
\end{tabular}

von der Gesamtproduktion von rund $152967 \mathrm{~kg}$ Feingold rund $48080 \mathrm{~kg}$, Australien $42980 \mathrm{~kg}$ und Rußland $35765 \mathrm{~kg}$ lieferte; der Rest mit 16\% kam auf die übrigen Länder zusammen.

Mit der 1886 gemachten Entdeckung der reichen Goldlager in Transvaal beginnt die Produktion zu steigen. Schon im Jahre 1888 lieferte Transvaal $6220 \mathrm{~kg}$, während es 1895 in dem berühmten Distrikte von Witwatersrand allein $57380 \mathrm{~kg}$ und $1898110778 \mathrm{~kg}$ produzierte. Es wïrde im folgenden Jahre $140000 \mathrm{~kg}$ überschritten haben, wenn nicht der im Oktober 1899 ausgebrochene Burenkrieg die Produktion unterbrochen hätte.

Aber auch die Goldproduktion der Vereinigten Staaten erfubr durch die Entdeckung der Goldlager in Colorado, besonders der von Cripple-Creek, eine Steigerung, die bis 1893 allerdings bascheiden war, von $1893 \mathrm{ab}$ aber eine bedeutende ist. 1893 lieferte Nordamerika $55083 \mathrm{~kg}, 1900$ aber $119113 \mathrm{~kg}$.

Eine weitere Steigerung der Goldgewinnung brachte die Entdeckung neuer Goldfelder in Westaustralien, wodurch die australische Goldproduktion im Jahro 1893 auf $54083 \mathrm{~kg}$ und 1899 auf $127697 \mathrm{~kg}$ stieg.

Die 1896 entdeckten Goldlager von Klondyke im Nordwesten von Kanada brachten die bis dahin unbedeutende Produktion Kanadas in Gold im Jahre 1896 auf $4230 \mathrm{~kg}$ und 1900 auf $41985 \mathrm{~kg}$.

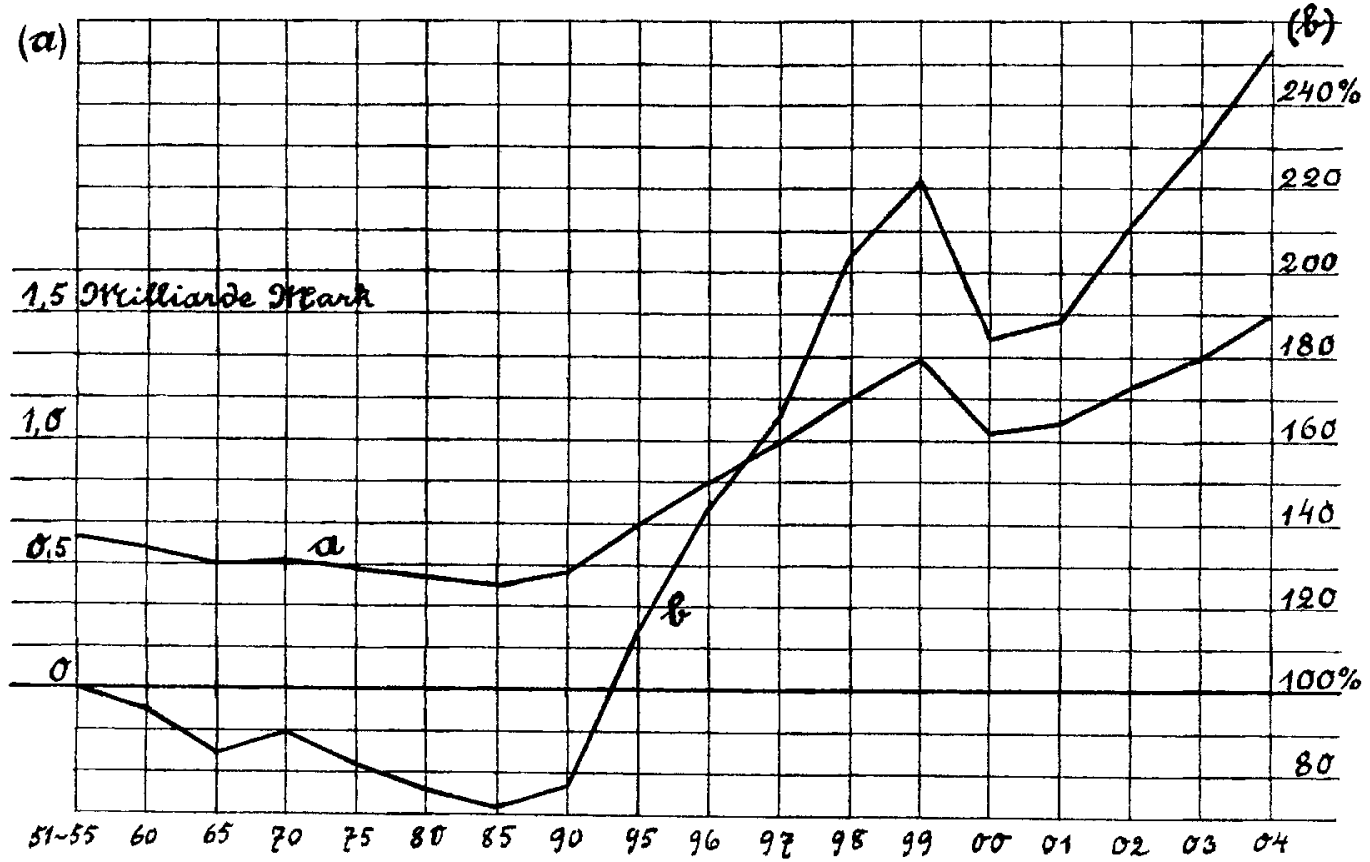

Fig. 1.

Das Schaubild der Reihen a und $b$ dieser Tabelle gibt Figur 1.

Wie dic Tabelle zeigt, war die Goldgewinnung in dem Zeitraume von $1880-1885$ am niedrigsten; sie betrug 1882418,98 Millionen $M$ und 1883 400,65 Millionen $M$ und entfiel zu $84 \%$ anf die damaligen Hauptproduzenten: die Vereinigten Staaten von Nordamerika, Rußland und Australasien (Australien und Neu-Seeland), indem Amerika
Ferner fallen in diese Zeit neue Goldfunde in Mexiko und Englisch-Indien, beziehungsweise wird seit dieser Zeit die Goldgewinnung auch in Mexiko und Englisch-Indien ausgedehnter betrieben, so daß Mexiko im Jahre $190013529 \mathrm{~kg}$ und EnglischIndien $15426 \mathrm{~kg}$ produzierte.

Seit Mitte der achtziger Jahre ist also auf der ganzen Erde die Goldgewinnung teils infolge Entdeckung neuer Goldlager, teils durch energischere 
Ausbeutung der bereits bekannten Lager ganz außerordentlich gestiegen und würde ohne das Dazwischentreten des Burenkrieges 1900 die Gesamtproduktion auf 1,5 Milliarden $M$ gekommen sein.

Die nachfolgende Tabelle gibt die Produktion der wichtigsten Produzenten.
Rußland, dessen Hauptfundstätten in Sibirien liegen, scheint, soweit die ziemlich unvollkommenen statistischen Angaben und der in großem Maßstabe betriebene Schmuggel ein Urteil möglich machen, in seiner Produktion seit Jahren sich gleich zu bleiben.

Mexiko und Englisch.Indien zeigen eine geringe

\begin{tabular}{|c|c|c|c|c|c|c|c|c|c|c|}
\hline \multirow{2}{*}{ Produzent } & \multicolumn{10}{|c|}{ Produktion in $\mathrm{kg}$ Feingold } \\
\hline & 1895 & 1896 & 1897 & 1898 & 1899 & 1900 & 1901 & 1902 & 1903 & 1904 \\
\hline 1. Aus & & 65985 & & 39995 & 127697 & 116003 & 117 & 122814 & 134290 & 130154 \\
\hline 2. Afrika & 65777 & 668 & 87515 & 121446 & 114 & 17 & 14 & 62 & 103190 & 129501 \\
\hline 3. Yor. Stratol r. N. A. & 70131 & 79865 & 86303 & 96970 & 106891 & 119113 & 118336 & 120357 & 110716 & 127199 \\
\hline 4. Kanada... & - & 4230 & $\ldots$ & - & 32095 & 41985 & 36791 & 31193 & 28332 & 25564 \\
\hline 5. Rußland & - & - & - & 一 & $\mathbf{3 3} 339$ & 323 & 35299 & 34210 & 37040 & 33899 \\
\hline 6. Mexiko.... & - & - & - & - & 13995 & 13529 & 15 & 15270 & 16048 & 16328 \\
\hline 7. Englisch-Indien & - & - & - & 一 & 13933 & 15426 & 15612 & 15985 & 18598 & 18847 \\
\hline Verschiedene. . . & & & & & $\mathrm{D}$ e $\mathrm{r}$ & 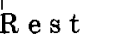 & & & & \\
\hline Im ganzen & 300177 & 305402 & 357152 & 435898 & 473.342 & 400102 & 401035 & 449022 & 490696 & 526399 \\
\hline
\end{tabular}

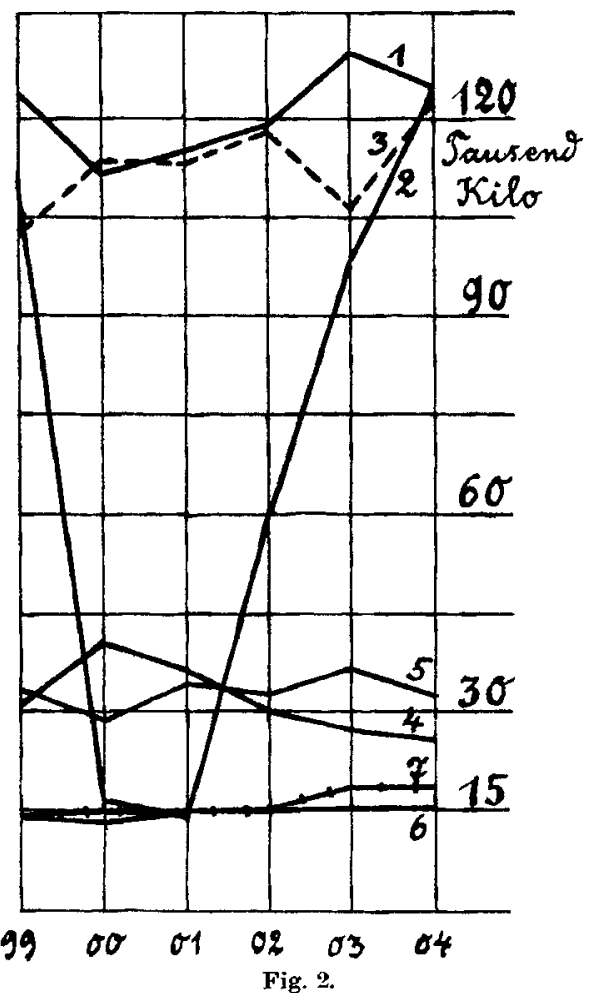

Prüft man diese Tabelle und das dieselbe darstellende Schaubild Fig. 2, so zeigt es sich, dal3 Australasien im Jahre 1900 gegen 1899 einen bedeutenden Abfall hatte, daß es jedoch nach einer leichten Zunahme im Jahre 1901 und 1902 im Jahre 1903 einen bedeutenden Produktionszuwachs erlebte und im Jahre 1904 ungefähr wieder auf die Höhe von $1899 \mathrm{kam}$.

Die Produktion der Vereinigten Staaten ist bei einigen Schwankungen zwischen 1900 und 1904 ziemlich dieselbe geblieben.

Die Produktion Kanadas hat seit 1900 stetig abgenommen, da die Lager von Klondyke bereits ausgebeutet zu sein scheinen, und die übrigen Fundstätten von geringerem Werte sind. stetige Produktionszunahme, während die übrigen Länder seit 1900 sich auf derselben Höhe halten.

Während nun aber die Goldproduktion der übrigen Produzenten in den letzten Jahren die nämliche geblieben ist, beziehungsweise nur im geringen Maße zunimmt, steigt die Produktion Afrikas nach dem Burenkriege in auBerordentlichem Maße. Seine Produktion war $1904129400,4 \mathrm{~kg}$ Feingold, von denen allein $113639 \mathrm{~kg}$, also im Monat durchschnittlich $9470 \mathrm{~kg}$, auf Witwatersrand entfallen. Im Dezember 1904 lieferte Witwatersrand $10885 \mathrm{~kg}$ Feingold und im Januar $190511103 \mathrm{~kg}$, so daß man für 1905 wohl auf eine monatliche Durchschnittsproduktion von wenigstens $12450 \mathrm{~kg}$, das heißt also auf eine Zunahme der Jahresproduktion Afrikas, herbeigeführt allein durch die Minen von Witwatersrand, von 38000 bis $49000 \mathrm{~kg}$ wird rechnen können. Es ist wahrscheinlich, daß diese Produktionszunahme noch mehrere Jahre andauern wird, und $\mathrm{daB}$ man, wie die südafrikanischen Goldkönige hoffen, bis 1910 vielleicht auf 220000 bis $250000 \mathrm{~kg}$ pro Jahr kommen wird.

Die übrigen afrikanischen Minen, wie die in Westafrika, an der Goldküste und an der Elfenbeinküste, haben noch nicht viel Erfolg erzielt. Sie ergaben, nachdem sie jetzt etwa 4 Jahre im Betriebe sind, im letzten Jahre einige hundert Kilo, während Witwatersrand vier Jahre nach seiner Entdeckung schon $12440 \mathrm{~kg}$ lieferte. Die RhodesiaMinen gaben $19026233,4 \mathrm{~kg}, 19037215,2 \mathrm{~kg}$ und $19048334,8 \mathrm{~kg}$, scheinen also auch keine große Entwicklung zu versprechen. Augenblicklich befaßt man sich in London viel mit den ägyptischen Goldlagern, die in der arabischen Wüste zwischen dem Roten Meere und dem unteren und mittleren Laufe des Niles sich befinden; bis jetzt hat man jedoch noch keine Resultate.

Untersucht man, aus welchen Gründen die Goldproduktion in den übrigen Staaten nicht zugenommen hat, so kommt man zu dem Schlusse, daß im allgemeinen nicht die Abnahme des Goldvorkommens in ihnen der Grund ist, sondern andere Ursachen vorliegen.

So hatte Nordamerika im Jahre 1903 und Anfang 1904 unter den großen Striken der Minen- 
arbeiter zu leiden, so daß seine Produktion im Jahre 1903 um $9641 \mathrm{~kg}$ niedriger war, als 1902 . In Colorado, dem Hauptproduzenten Amerikas, war die Gewinnung von $42824,7 \mathrm{~kg}$ im Jahre 1902 auf $33899 \mathrm{~kg}$ im Jahre 1903 gefallen und lieferte auch 1904 noch nicht mehr als $39123,8 \mathrm{~kg}$. In diesem Jahre dürfte es aber seine alte Höhe wieder erreichen, wenn nicht überschreiten, trotz des Eingehens mehrerer Fundstätten, die aber durch neuentdeckte Goldlager ausgeglichen werden. Kalifornien lieferte $190428652 \mathrm{~kg}$ gegen $24.226,9 \mathrm{~kg} \mathrm{1903 \text {; }}$ Nevada $19047743,9 \mathrm{~kg}$ gegen $5100,4 \mathrm{~kg}$ im Jahre 1903; Utah $19047059,7 \mathrm{~kg}$ gegen $5566,9 \mathrm{~kg} 1903$. Auch die Ausbeutung in Alaska ist 1904 größer als 1903. Da außerdem die Unternehmungslust in den Vereinigten Staaten augenblicklich sehr rege ist, so ist in den Verein. Staaten für 1905, wenn nicht neue Arbeitseinstellungen stattfinden, eine erhebliche Produktionszunahme zu erwarten.

Für Australien sind die Aussichten vielleicht weniger gut. Nach einem bedeutenden Rückgange im Jahre 1900 hat Westaustralien seine Produktion wieder gesteigert, bis es 1904 abermals eine wenn auch nur geringe Abnahme (von $75759,6 \mathrm{~kg} 1903$ auf $73773,2 \mathrm{~kg}$ 1904) aufweist. Queensland hat einen Rückgang von $28301 \mathrm{~kg} 1903$ auf $25782 \mathrm{~kg}$ 1904. Viktoria und Neuseeland haben sich ziemlich auf ihrer Höhe gehalten. Tasmanien und Südaustralien gewinnen fast gar kein Gold mehr, obwohl man vor etwa 2 Jahren viel von der Entdeckung eines Goldlagers in Arltunga sprach. Die australischen Minen sind vielfach schlecht verwaltet. Die Gewinnung ist oft, z. B. in Queensland, eine sehr primitive und sehr wenig wissenschaftliche. Es scheint, $\mathrm{da} \beta$ für 1905 bei den australischen Minen eine große Entwicklung nicht zu erwarten ist.

Betreffend die übrigen Länder ist eine große Änderung ebenfalls nicht zu erwarten; vor allem gewiß nicht in Sibirien.

Die sicher zu erwartende Zunahme der Produktion von Afrika und Nordamerika genügt jedoch, die Goldproduktion von 1905 auf die Höhe von 1600 Millionen zu bringen, also auf das Doppelte der Produktion im Jahre 1896, Zehn Jahre früher (1885) war die Produktion 426 Millionen Mark. Wird man nun nach zehn Jahren, 1915, die Produktion abermals verdoppelt, also auf 3200 Millionen Mark gebracht sehen?

Diese Frage ist schwer zu beantworten. Doch ist es kaum zu bezweifeln, daß fortgesetzt neue und ausgiebige Goldlager entdeckt werden. Australien birgt zweifellos noch reiche Fundstätten, ebenso Afrika. In Amerika sind die Schätze der Felsengebirge noch lange nicht erschlossen und ausgebeutet. Andererseits werden auch die südamerikanischen Staaten die reichen Schätze der Anden, die bis heute nur oberflächlich ausgebeutet werden, zu heben sich bemühen. Auch Rußland wird durch liberale und günstige Maßnahmen die vortcilhaftere Ausbeutung seiner sibirischen Goldlager möglich machen. Alles dieses läßt auch für die folgenden Jahre eine Produktionssteigerung erwarten und macht eine bedeutende Zunahme nicht unwahrscheinlich.

$\mathrm{Ob}$ aber eine solche Steigerung für die Volkswirtschaft vorteilhaft sein wird, ist $\mathrm{zu}$ bezweifeln.
Nach statistischen Berechnungen war die gesamte Goldproduktion seit der Entdeckung Amerikas bis 1885 rund 30 Milliarden Mark; seit 1885 bis einschließlich 1904 betrug sie aber etwas mehr als 16 Milliarden Mark. Da vor 1492 nur einige hundert Millionen Mark an Gold vorhanden waren, so ist der gesamte Goldvorrat der Welt seit 20 Jahren um mehr als die Hälfte gestiegen, nämlich von rund 30 Milliarden Mark auf 46 Milliarden Mark. Unter der Annahme, daß bis 1915 die Jahresproduktion auf 3,2 Milliarden Mark steigt, wïrde 1915 der Goldvorrat rund 70 Milliarden Mark betragen. Dieses muß aber die schwersten Störungen des Goldmarktes zur Folge haben.

\section{Bekanntmachung betr. Sammlung chemischer Analysen von Gesteinen, Bodenarten und Grundwässern.}

In den chemischen Laboratorien der Universitäten und Hochschulen wie insbesondere der LandwirtschaftlichenVersuchsstationen, technisohen Laboratorien usw. werden alljährlich viele Tausende chemischer Analysen von deutschen Gesteinen, Bodenarten, Brunnen-, Quell-, Grund- und Seewässern ausgeführt, um bestimmte örtliche Einzelfragen der Praxis zu beantworten. Nur ein ver. schwindend kleiner Teil dieser Analysen wird veröffentlicht. Die meisten verfallen, sobald sie den praktischen Zweck, der sie veranlaßte, erfüllt haben, der Vergessenheit, mögen sie auch noch so genau und zuverlässig ausgeführt worden sein. Das gewaltige Kapital von geistiger Arbeit, welches in dicsen Analysen der Praxis niedergelegt ist, könnte aber für die Wissenschaft wie für die Praxis noch weitere Früchte tragen, wenn es gelänge, die Ergebnisse dieser Analysen nach einheitlichen Gesichtspunkten zusammenzufasson.

Dies ist leider nicht in allen Fällen möglich, da die Herkunft und geologische Benennung der untersuchten Proben, wie die Art der Probeentnahme auch den ausführenden Chemikern nicht immer so genau bekannt sind, daB danach das Einzelergebnis irgendwie verallgemeinert werden könnte. Wo aber diese Vorbedingungen erfüllt sind, ist es dringend erwünscht, daß die Analysen aufbewahrt, gesammelt und, nach geologischen Gesichtspunkten geordnet, der Algemeinheit zugänglich gemacht werden.

Die Kgl. Preussische Geologische Landesanstalt und Bergakademie wird sich dieser mühsamen, aber gemeinnützigen Aufgabe unterziehen. Selbst wenn nur wenige Bruchteile der zu praktischen Zwecken unternommenen Analysen auf solche Weise gerettet werden, gewinnt die deutsche Landwirtsohaft, wie jedes sonstige, auf Bodennutzung angewiesene Gewerbe schon nach wenigen Jahren ein reiches und mit der Zeit immer vielseitiger, eingehender und durch Ableitung von Mittel- und Grenzzahlen immer zutreffender werdendes Bild von der chemischen Zusammensetzung wie von den wirtschaftlichen Eigenschaften jedes einzelnen, auf der geologischen Karte unterschiedenen Gesteins wie der daraus hervorgegangenen Bodenarten.

Zwar bieten bereits die in unserem Boden- 